\title{
Breast cancer treatment and survival differences in women in remote and socioeconomically disadvantaged areas, as demonstrated by linked data from New South Wales (NSW), Australia
}

\author{
Elizabeth Buckley ${ }^{1,3} \cdot$ Elisabeth Elder $^{2} \cdot$ Sarah McGill ${ }^{3} \cdot$ Zahra Shahabi Kargar $^{3} \cdot$ Ming Li $^{1} \cdot$ David Roder $^{1,3}$ (D) \\ David Currow ${ }^{3}$
}

Received: 13 January 2021 / Accepted: 24 February 2021 / Published online: 21 March 2021

(c) The Author(s) 2021

\begin{abstract}
Introduction Reducing variations in cancer treatment and survival is a key aim of the NSW Cancer Plan. Variations in breast cancer treatment and survival in NSW by remoteness and socioeconomic status of residence were investigated to determine benchmarks. Reducing variations in cancer treatment and survival is a key aim of the NSW Cancer Plan. Variations in breast cancer treatment and survival in NSW by remoteness and socioeconomic status of residence were investigated to determine benchmarks.

Methods A retrospective cohort study used linked data for invasive breast cancers, diagnosed in May 2002 to December 2015 from the NSW Cancer Registry, with corresponding inpatient, and medical and pharmaceutical insurance data. Associations between treatment modalities, area socioeconomic status and residential remoteness were explored using logistic regression. Predictors of breast cancer survival were investigated using Kaplan-Meier product-limit estimates and multivariate competing risk regression.

Results Results indicated a high 5-year disease-specific survival in NSW of 90\%. Crude survival was equivalent by residential remoteness and marginally lower in lower socioeconomic areas. Competing risk regression showed equivalent outcomes by area socioeconomic status, except for the least disadvantaged quintile, which showed a higher survival. Higher sub-hazard ratios for death occurred for women with breast cancer aged $70+$ years, and more advanced stage. Adjusted analyses indicated more advanced stage in lower socioeconomic areas, with less breast reconstruction and radiotherapy, and marginally less hormone therapy for women from these areas. Conversely, among these women who had breast conserving surgery, there was higher use of chemotherapy. Remoteness of residence was associated in adjusted analyses with less radiotherapy and less immediate breast reconstruction. In these short term data, remoteness of residence was not associated with lower survival. Conclusion This study provides benchmarks for monitoring future variations in treatment and survival.
\end{abstract}

Keywords Breast cancer survival $\cdot$ New South Wales $\cdot$ Breast cancer treatment $\cdot$ Socioeconomic status $\cdot$ Residential remoteness

David Roder

David.roder@unisa.edu.au

1 Cancer Epidemiology and Population Health Research Group, Allied Health \& Human Performance, University of South Australia, Adelaide, Australia

2 Specialist Breast Surgery, Westmead Breast Cancer Institute, Westmead, NSW 2145, Australia

3 Cancer Institute NSW, Level 4, 1 Reserve Road, St Leonards, NSW 2065, Australia

$\begin{array}{ll}\text { Abbreviations } \\ \text { APDC } & \text { Admitted Patient Data Collection } \\ \text { ARIA } & \text { Accessibility Remoteness Index of Australia } \\ \text { BCS } & \text { Breast conserving surgery } \\ \text { CI } & \text { Confidence interval } \\ \text { HER2 } & \text { Human Epidermal Growth Factor receptor } 2 \\ \text { ICD-O-3 } & \text { International Classification of Diseases for } \\ & \text { Oncology, third version } \\ \text { IRSD } & \text { Index of Relative Socioeconomic Disadvantage } \\ \text { LHDs } & \text { Local health districts } \\ \text { MBS } & \text { Medicare Benefits Schedule } \\ \text { PBS } & \text { Pharmaceutical Benefits Schedule } \\ \text { NSW } & \text { New South Wales }\end{array}$


NSWCR New South Wales Cancer Registry

SURE Secure Unified Research Environment

\section{Introduction}

Breast cancer is the leading cancer reported in women by Australian cancer registries and second to lung cancer as the leading cause of cancer death in women [1]. Substantial increases in survival from breast cancer in Australia have been recorded, largely attributed to advances in systemic therapies and population-based screening [2]. However, as for many chronic diseases, survival estimates vary across the population [2].

Increased socioeconomic disadvantage and geographical remoteness have been associated with poorer health in many countries, including in Australia [3]. Australia has many geographically remote areas where there may be barriers to accessing health services. Residents living remotely have been found to be diagnosed with breast cancer at a later stage than those in major cities [4]. Also, women living in areas of socioeconomic disadvantage have been less likely to participate in screening and more likely to experience poorer outcomes [4-7].

A principal aim of the NSW Cancer Plan is to improve survival and reduce variation across the NSW population [8]. A range of interventions is used, including provision of ongoing feedback to services from health-service monitoring with linked data [9]. Feedback indicates differences in clinical practice and outcomes that warrant further exploration. Evaluation studies are undertaken regularly to quantify trends in variations and inform planning cycles.

This study is one such evaluation. It investigates invasive breast cancer treatment and survival across areas of differing socioeconomic status and residential remoteness using linked population-based cancer registry notifications. Treatment categories studied included: treatment by surgery; treatment by surgery type (breast conserving and mastectomy); adjuvant radiotherapy following breast conserving surgery (a guideline recommendation); use of adjuvant chemotherapy or hormone therapy where clinically indicated; and reconstruction following mastectomy.

\section{Methods}

Data on histologically confirmed breast cancers (ICD-O-3 C50) from the NSW Cancer Registry (NSWCR) were linked to the NSW Admitted Patient Data Collection (APDC), health insurance claims data from the Medicare Benefits (MBS) and Pharmaceutical Benefits (PBS) schemes, and data from the National Death Index to include deaths occurring outside NSW, as described previously $[10,11]$.
Notification to the NSWCR of new cancers affecting NSW residents is mandatory under the Public Health Act [12]. Sources of notifications include pathology laboratories, public and private hospitals, imaging centres, aged care facilities and official death registrations from the NSW Registry for Births, Deaths and Marriages [13]. In NSW and other Australian States and Territories, the underlying cause of death is determined by the medical practitioner certifying the death[14].

Linked APDC records include women's demographic descriptors and information on cancer, other diagnoses and in-hospital procedures performed in NSW public and private hospitals. Principal and additional procedures were identified from Australian Classification of Health Interventions procedural codes [14]. Linked medical claims data were obtained for medical services and procedures, and publicly funded dispensed medications, respectively $[15,16]$.

For this study, female breast cancers diagnosed from 1st May 2002 until 31st December 2015 were included. Some were excluded where breast cancer surgery (breast conserving or mastectomy) preceded cancer diagnosis by more than 30 days. Also, women from five local health districts adjacent to the NSW border (12\%) were excluded for the analysis of treatment only, because NSW information systems did not cover treatment provided outside NSW.

Data were linked as described in the NSW Cancer Plan [8]. Linkage was performed by the Centre for Health Record Linkage for NSW-based datasets and by the Australian Institute of Health and Welfare for linkage to Commonwealthbased datasets [11]. Data were stored in the SURE facility, a remote access computing environment to which authorized analysts were given encrypted access with strong authentication [17].

Demographic data were classified by age at diagnosis ( $<40,40-49,50-59,60-69,70-79,80+$ years) and country of birth (Australia, other mainly English speaking and mainly non-English speaking countries)[10]. Additionally, the Index of Relative Socioeconomic Disadvantage (IRSD) and Accessibility/Remoteness Index of Australia (ARIA) were used as area-level indices of socioeconomic disadvantage and remoteness of residence, respectively $[18,19]$.

First surgery after diagnosis was identified and classified as whether performed within 12 months following diagnosis (or in the 30 days prior to diagnosis to allow for delays in notification), using APDC procedural codes from the Australian Classification of Health Interventions (ACHI 8th edition) [20]. Breast surgeries identified from MBS item numbers, were classified as breast conserving surgery or reexcision, and mastectomy, and used to supplement APDC records [15]. Breast surgery performed within 12 months of diagnosis was classified as breast conserving (including 
re-excisions) or mastectomy. Women were also classified as having or not having breast surgery within this period.

Radiotherapy was identified from APDC data, plus radiotherapy centre and MBS records, and classified as whether occurring within 12 months of diagnosis [10]. Procedure codes for chemotherapy delivery were obtained from admission records, MBS items, and also PBS items classified using WHO Anatomical Therapeutic Clinical Classification System (ATC) codes, where identified as occurring within 12 months of diagnosis or not [16]. Specific codes for targeted and hormone therapy were identified in PBS data and dates of supply were used to indicate administration beginning within 12 months of diagnosis.

Clinical care and survival were analysed by level of socioeconomic disadvantage (Quintiles 1-2 versus 3-5) and of residential remoteness (remote/very remote versus major city/inner regional/outer regional) [18, 19].

\section{Statistical analyses}

Descriptive statistics were calculated for all women, including those residing in border local health districts, across study variables, collapsing rows or columns as needed to avoid cell sizes $<5$. Descriptive statistics for treatment variables were calculated for NSW residents who resided outside of local health districts adjacent to the NSW border. Crude associations were calculated by logistic regression, and then with adjustment for sociodemographic (i.e., age, country of birth, diagnostic period, histology type, extent of disease, and residential IRSD and remoteness ARIA) and clinical factors. Clinical exposures examined were any treatment, any surgery, type of surgery (breast conserving or mastectomy), radiotherapy following breast conserving surgery, and reconstruction following mastectomy. Penalized logistic regression was used to model associations between study variables and remoteness to avoid issues of sparse data and separation [21, 22]. Crude and adjusted logistic regressions involving treatment variables (including penalized logistic regression) excluded those women in border local health districts $(n=6447)$.

Disease-specific survivals at 1 year, 2 year, 3 year and 5 years following diagnosis were calculated using Kaplan Meier product-limit estimates [23] for all women including those living in border local health districts. These estimates have been shown to be good proxies for relative survival in Australia where causes of death are assigned by registry staff based on cancer notifications, hospital reporting and death records [24]. This is important, given the uncertain accuracy of causes of death recorded on death registrations in many international studies [25]. Disease-specific survival was chosen because life tables were not available for many comparison groups to use net survival [23].

In this study, survival times were measured from diagnosis until death or censoring on December 31, 2015, whichever occurred first. Corresponding measures of breast cancer survival were obtained using competing risk regression (competing deaths being those from causes other than breast cancer) [26]. Predictors included age at diagnosis, residential remoteness, socioeconomic status, country of birth, diagnostic period, histology type and stage (extent of disease). The proportional hazards assumption was tested, and for variables where it was found not to be met, interaction terms with natural log time (years) were included.

All analyses were undertaken using Stata 16.0 [23].

\section{Results}

\section{Descriptive characteristics}

There were 62,681 women initially included in the linked dataset (Fig. 1). After excluding those of unknown socioeconomic status $(n=2)$, those having prior breast surgery $(n=1472)$, those living in a border local health district $(n=6,447)$ and those with multiple primaries $(n=6545)$, 48,215 were available for analysis of treatment variables, and 54,662 for survival analyses.

Approximately half (51\%) these women were aged from 50 to 69 years (Table 1). Over 70\% lived in major city areas and $43 \%$ in areas of socioeconomic advantage (IRSD $\mathrm{Q} 4 / 5)$. The proportion living in areas of greatest socioeconomic disadvantage (Q1) comprised $17 \%$. Approximately

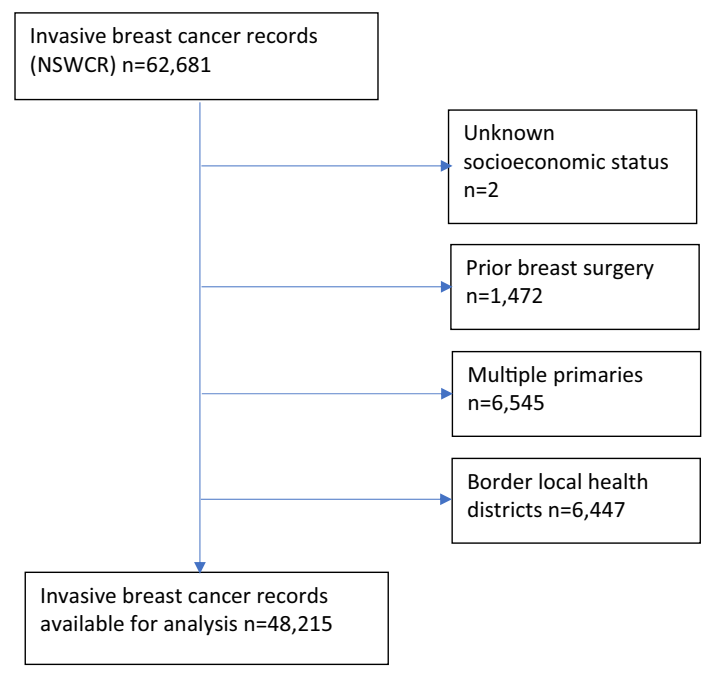

$\underline{\text { NSWCR - New South Wales Cancer Registry }}$

Fig. 1 Application of inclusion/exclusion criteria for analysis 
Table 1 Descriptive statistics for women with invasive breast cancer, NSW 2002-2015

\begin{tabular}{|c|c|}
\hline Variable & $\mathrm{N}(\%)$ \\
\hline All & $54,662(100)$ \\
\hline \multicolumn{2}{|l|}{ Age group (years) } \\
\hline$<40$ & $3,212(5.9)$ \\
\hline $40-49$ & $10,177(18.6)$ \\
\hline $50-59$ & $14,194(26.0)$ \\
\hline $60-69$ & $13,855(25.4)$ \\
\hline $70-79$ & $8,255(15.1)$ \\
\hline $80+$ & $4,969(9.1)$ \\
\hline \multicolumn{2}{|l|}{ Remoteness } \\
\hline Metropolitan & $38,670(70.7)$ \\
\hline Inner regional & $12,148(22.2)$ \\
\hline Outer regional & $3,596(6.6)$ \\
\hline Remote \& very remote & $248(<1)$ \\
\hline \multicolumn{2}{|l|}{ Socioeconomic status (quintiles) } \\
\hline 1 (Most disadvantaged) & $9,304(17.0)$ \\
\hline 2 & $11,221(20.5)$ \\
\hline 3 & $10,702(19.6)$ \\
\hline 4 & $10,665(19.5)$ \\
\hline 5 (Least disadvantaged) & $12,770(23.4)$ \\
\hline \multicolumn{2}{|l|}{ Country of Birth } \\
\hline Australia & $36,517(66.8)$ \\
\hline Other English-speaking countries & $5,741(10.5)$ \\
\hline Non-English-speaking countries & $12,404(22.7)$ \\
\hline \multicolumn{2}{|l|}{ Diagnosis Year } \\
\hline $2002-2007$ & $19,805(36.2)$ \\
\hline $2008-2015$ & $34,857(63.8)$ \\
\hline \multicolumn{2}{|l|}{ Histology } \\
\hline Ductal & $40,596(74.3)$ \\
\hline Lobular & $5,778(10.6)$ \\
\hline Other & $8,288(15.2)$ \\
\hline \multicolumn{2}{|l|}{ Extent of disease } \\
\hline Local & $28,158(51.5)$ \\
\hline Regional & $20,523(37.6)$ \\
\hline Distant & $3,173(5.8)$ \\
\hline Unknown & $2,808(5.1)$ \\
\hline \multicolumn{2}{|c|}{$\begin{array}{l}\text { In women residing outside of border local health } \\
\text { districts }(n=48,215)\end{array}$} \\
\hline \multicolumn{2}{|l|}{ Surgery within 12 months } \\
\hline None & $3,845(8.0)$ \\
\hline Breast conserving & $25,517(52.9)$ \\
\hline Mastectomy & $18,853(39.1)$ \\
\hline \multicolumn{2}{|c|}{ Reconstruction following mastectomy $(n=18,853)$} \\
\hline Immediate & $1,043(5.5)$ \\
\hline Within 12 months & $852(4.5)$ \\
\hline Within 5 years & $681(3.6)$ \\
\hline None & $16,277(86.3)$ \\
\hline \multicolumn{2}{|l|}{ Radiotherapy within 12 months } \\
\hline Yes & $28,869(59.9)$ \\
\hline No & $19,346(40.1)$ \\
\hline
\end{tabular}

Table 1 (continued)

\begin{tabular}{ll}
\hline Variable & $\mathrm{N}(\%)$ \\
\hline Chemotherapy within 12 months & \\
Yes & $21,272(44.1)$ \\
No & $26,943(55.9)$ \\
Hormone therapy within 12 months & \\
Yes & $32,116(66.6)$ \\
No & $16,099(33.4)$ \\
Immunotherapy within 12 months & \\
Yes & $3,843(8.0)$ \\
No & $44,372(92.0)$ \\
\hline
\end{tabular}

two thirds (67\%) were born in Australia, $11 \%$ in other mainly English-speaking countries and 23\% in mainly non-English speaking countries. More than half (52\%) of those with known stage were diagnosed with local disease and approximately $6 \%$ with distant disease. The percentage treated for breast cancer within 12 months of diagnosis was $92 \%$ for breast surgery ( $53 \%$ for breast conserving surgery and 39\% for mastectomy), $60 \%$ for radiotherapy, $44 \%$ for chemotherapy, $67 \%$ for hormone therapy, and $8 \%$ for immunotherapy. Five percent had breast reconstruction (i.e., $14 \%$ of those having a mastectomy).

\section{Unadjusted comparisons by socioeconomic status and remoteness}

\section{Socioeconomic status}

Women aged 50-79 years at diagnosis had 11-29\% higher odds of living in areas of greater socioeconomic disadvantage than those aged $<40$ years (Table 2 ). Living in inner regional, very remote and, more so, outer regional areas was strongly associated with socioeconomic disadvantage (ORunadj 39.4 [95\% CI 32.9, 47.1] for outer regional). Women who were born in other mainly English-speaking countries had 27\% lower odds than women born in Australia of living in areas of greater socioeconomic disadvantage (ORunadj 0.73 [95\% CI 0.68, 0.78]), whereas those born in other mainly non-English-speaking countries had $16 \%$ higher odds of living in areas of socioeconomic disadvantage.

Women with cancers of non-ductal histology type were less likely to live in areas of greater socioeconomic disadvantage (36\% and 38\%, respectively; ORunadj 0.89 [95\% CI $0.84,0.95]$ ). Being diagnosed with distant disease was also more likely to occur in women living in socioeconomically disadvantaged areas (ORunadj 1.31 [95\% CI 1.21, 1.42]). Women undergoing any surgery (breast conserving or mastectomy) were less likely to live in areas of greater socioeconomic disadvantage, as were those receiving radiotherapy, 
Table 2 Unadjusted logistic regression on socioeconomic disadvantage (Quintile 1 and Quintile 2) and remoteness (remote and very remote), respectively
Independent variables

Age group (years)

$<40$

$40-49$

$50-59$

$60-69$

$70-79$

$80+$

Remoteness

Metropolitan

Inner regional

Outer regional

Remote \& very remote

Socioeconomic status (quintiles)

1 (Most disadvantaged)

2

3

4

5 (Least disadvantaged)

Country of Birth

Australia

Other English-speaking countries

Non-English-speaking countries

Diagnosis Year

2002-2007

2008-2015

Histology

Ductal

Lobular

Other

Extent of disease

Local

Regional

Distant

Unknown

Any treatment within 12 months

No

Yes

Any surgery within 12 months

No

Yes

Surgery within 12 months

None

Breast conserving

Mastectomy

Reconstruction $^{\text {b }}$

Immediate

Within 12 months

Within 5 years

None

Odds Ratio [95\% CI] $(\mathrm{n}=48,215)$

Socioeconomic disadvantage

Remote \& very remote ${ }^{\mathrm{a}}$ (Q1\&Q2)

1.0 (reference)

$1.03[0.94,1.13]$

$1.11[1.02,1.21]$

$1.24[1.13,1.35]$

$1.29[1.18,1.41]$

$1.08[0.97,1.19]$

1.0 (reference)

3.59 [3.41, 3.77]

39.36 [32.90, 47.09]

141.27 [52.50, 380.14]

1.0 (reference)

$0.73[0.68,0.78]$

$1.16[1.11,1.21]$

1.0 (reference)

$0.96[0.92,0.99]$

1.0 (reference)

$0.89[0.84,0.95]$

$0.93[0.88,0.98]$

1.0 (reference)

$1.00[0.96,1.05]$

$1.31[1.21,1.42]$

$1.02[0.93,1.12]$

1.0 (reference)

$0.95[0.82,1.09]$

1.0 (reference)

$0.86[0.80,0.92]$

1.0 (reference)

0.83 [0.77, 0.89]

0.90 [0.84, 0.97]

$0.38[0.33,0.45]$

$0.35[0.29,0.42]$

$0.53[0.44,0.63]$

1.0 (reference)
1.0 (reference)

$1.57[0.75,3.28]$

$1.66[0.71,3.40]$

$1.44[0.69,2.97]$

$1.70[0.81,3.61]$

$1.07[0.45,2.53]$

1.0 (reference)

$0.23[0.17,0.32]$

$<0.01[<0.01,0.04]$

$0.02[<0.01,0.05]$

$<0.01[<0.01,0.03]$

1.0 (reference)

$0.30[0.16,0.58]$

$0.15[0.08,0.27]$

1.0 (reference)

$0.92[0.70,1.22]$

1.0 (reference)

$0.72[0.43,1.19]$

$0.95[0.65,1.39]$

1.0 (reference)

$0.91[0.68,1.23]$

1.14 [0.66, 1.97]

$1.0[0.53,1.89]$

1.0 (reference)

$0.64[0.28,1.51]$

1.0 (reference)

$0.79[0.50,1.25]$

1.0 (reference)

$0.75[0.47,1.21]$

$0.85[0.52,1.38]$

$0.10[0.01,1.55]$

$0.11[0.01,1.84]$

$0.72[0.20,2.53]$

1.0 (reference) 
Table 2 (continued)

\begin{tabular}{|c|c|c|}
\hline \multirow[t]{2}{*}{ Independent variables } & \multicolumn{2}{|c|}{ Odds Ratio $[95 \% \mathrm{CI}](\mathrm{n}=48,215)$} \\
\hline & $\begin{array}{l}\text { Socioeconomic disadvantage } \\
\text { (Q1\&Q2) }\end{array}$ & Remote \& very remote $^{a}$ \\
\hline \multicolumn{3}{|l|}{ Radiotherapy after BCS } \\
\hline No & 1.0 (reference) & 1.0 (reference) \\
\hline Yes & $0.85[0.80,0.91]$ & $0.73[0.47,1.15]$ \\
\hline \multicolumn{3}{|c|}{ Chemotherapy within 12 months } \\
\hline No & 1.0 (reference) & 1.0 (reference) \\
\hline Yes & $0.99[0.95,1.03]$ & $1.12[0.85,1.47]$ \\
\hline \multicolumn{3}{|c|}{ Hormone therapy within 12 months } \\
\hline None & 1.0 (reference) & 1.0 (reference) \\
\hline Yes & $0.94[0.91,0.98]$ & $1.04[0.78,1.40]$ \\
\hline \multicolumn{3}{|c|}{ Immunotherapy within 12 months } \\
\hline None & 1.0 (reference) & 1.0 (reference) \\
\hline Yes & $1.01[0.94,1.08]$ & $1.06[0.65,1.73]$ \\
\hline
\end{tabular}

hormone therapy, and breast reconstruction after mastectomy (Table 2).

\section{Residential remoteness}

Women living in more advantaged areas were less likely to live in more remote areas (Table 2). Those born outside of Australia were less likely to live in more remote areas compared with women born in Australia, especially if born in mainly non-English speaking countries with ORunadj 0.30 [95\% CI 0.16, 0.58] and (ORunadj 0.15 [95\% CI 0.08, 0.27]) respectively.

Although not reaching statistical significance, women undergoing reconstruction after mastectomy were less likely to reside in more remote areas (Table 2). Similarly, any treatment, or treatment by radiotherapy after breast conserving surgery was less common for residents of more remote areas at ORunadj 0.64 [0.28, 1.51and ORunadj $0.73[0.47,1.15]$, respectively (Table 2 ).

\section{Adjusted comparisons by socioeconomic status and remoteness}

\section{Socioeconomic status}

Five multivariate logistic regression models were used to predict the odds of socioeconomic disadvantage. All included age at diagnosis, residential remoteness, country of birth, histology type, stage, and diagnostic year, plus selected treatments, i.e.: any treatment (Model 1), and in addition to radiotherapy, chemotherapy, hormone therapy and immunotherapy, any surgery (Model 2), surgery type (Model 3), all variables limited to women having breast conserving surgery (Model 4); and breast reconstruction in women having a mastectomy (Model 5).

From Table 3, it is evident that odds of residing in a socioeconomically disadvantaged area were related to:

- Age at diagnosis - compared with $<40$ years, (a) elevated odds for 70-79 years in Models 1-4 and 60-69 years in Models 2-4; and (b) lower odds in Model 5 for ages 40-59 and 80+ years.

- Residential remoteness - compared with metropolitan areas, progressively higher odds for more remote areas in all Models.

- Country of birth - compared with Australia, elevated odds for mainly non-English speaking countries in all Models, and lower odds for mainly English-speaking countries in all Models.

- Diagnosis year - compared with 2002-2007, lower odds for 2008-2015 in Models 1-3.

- Histology type - compared with ductal, lower odds for lobular in Models 1-3 and 5.

- Stage (extent of disease) - compared with local disease, elevated odds for distant in all Models and for regional in Models 1-3.

- Breast reconstruction (mastectomy cases) - compared with no reconstruction, lower odds for immediate reconstruction and delayed reconstruction (12 month) in Model 5.

- Radiotherapy - lower odds for Models 2 and 4. 
Table 3 Association of sociodemographic, clinical and treatment factors with living in socioeconomically disadvantaged (IRSD Quntiles 1-2) areas in NSW

\begin{tabular}{|c|c|c|c|c|c|}
\hline \multirow[t]{2}{*}{ Independent variables } & \multicolumn{5}{|c|}{ Odds Ratio $[95 \% \mathrm{CI}]$ Socioeconomic disadvantage } \\
\hline & $\begin{array}{l}\text { Model } 1 \text { (any treat- } \\
\text { ment) }\end{array}$ & Model 2 (any surgery) & $\begin{array}{l}\text { Model } 3 \text { (surgery } \\
\text { type) }\end{array}$ & Model 4 (BCS cases) & $\begin{array}{l}\text { Model } 5 \text { (Mastectomy } \\
\text { cases) }^{\mathrm{a}}\end{array}$ \\
\hline \multicolumn{6}{|l|}{ Age group (years) } \\
\hline$<40$ & 1.0 (reference) & 1.0 (reference) & 1.0 (reference) & 1.0 (reference) & 1.0 (reference) \\
\hline $40-49$ & $0.96[0.88,1.06]$ & $0.97[0.88,1.07]$ & $0.97[0.88,1.07]$ & $1.00[0.87,1.16]$ & $0.86[0.75,0.98]$ \\
\hline $50-59$ & $1.01[0.92,1.11]$ & $1.02[0.93,1.12]$ & $1.03[0.94,1.13]$ & $1.05[0.91,1.21]$ & $0.85[0.74,0.98]$ \\
\hline $60-69$ & $1.09[0.99,1.19]$ & $1.11[1.01,1.22]$ & $1.11[1.01,1.22]$ & $1.09[0.94,1.26]$ & $0.93[0.81,1.08]$ \\
\hline $70-79$ & $1.15[1.04,1.27]$ & $1.17[1.06,1.29]$ & $1.17[1.06,1.30]$ & $1.14[0.98,1.33]$ & $0.93[0.80,1.08]$ \\
\hline $80+$ & $1.00[0.89,1.11]$ & $1.00[0.89,1.12]$ & $1.00[0.89,1.12]$ & $1.00[0.83,1.20]$ & $0.73[0.61,0.87]$ \\
\hline \multicolumn{6}{|l|}{ Remoteness } \\
\hline Metropolitan & 1.0 (reference) & 1.0 (reference) & 1.0 (reference) & 1.0 (reference) & 1.0 (reference) \\
\hline Inner regional & $4.22[4.00,4.44]$ & $4.21[4.00,4.44]$ & $4.21[4.00,4.43]$ & $4.05[3.78,4.35]$ & $4.56[4.20,4.95]$ \\
\hline Outer regional & $47.33[39.52,56.68]$ & $47.11[39.4,56.42]$ & $47.10[39.33,56.40]$ & $43.82[34.30,55.97]$ & $48.99[36.79,65.24]$ \\
\hline Remote/very remote & $\begin{array}{l}168.29[62.52 \\
453.01]\end{array}$ & $167[62.18,450.56]$ & $\begin{array}{l}167.37[62.17, \\
450.53]\end{array}$ & $112.35[35.59,54.66]$ & $522.51[32.41,8423.96]$ \\
\hline \multicolumn{6}{|l|}{ Country of Birth } \\
\hline Australia & 1.0 (reference) & 1.0 (reference) & 1.0 (reference) & 1.0 (reference) & 1.0 (reference) \\
\hline $\begin{array}{l}\text { Other English-speak- } \\
\text { ing countries }\end{array}$ & $0.87[0.81,0.93]$ & $0.87[0.81,0.93]$ & $0.87[0.81,0.93]$ & $0.87[0.79,0.96]$ & $0.86[0.77,0.97]$ \\
\hline $\begin{array}{l}\text { Non-English-speaking } \\
\text { countries }\end{array}$ & $1.89[1.80,1.98]$ & $1.88[1.79,1.97]$ & $1.88[1.79,1.97]$ & $1.90[1.77,2.03]$ & $1.83[1.69,1.97]$ \\
\hline \multicolumn{6}{|l|}{ Diagnosis Year } \\
\hline 2002-2007 & 1.0 (reference) & 1.0 (reference) & 1.0 (reference) & 1.0 (reference) & 1.0 (reference) \\
\hline 2008-2015 & $0.94[0.91,0.98]$ & $0.95[0.91,0.99]$ & $0.95[0.91,0.99]$ & $0.96[0.90,1.02]$ & $0.99[0.93,1.07]$ \\
\hline \multicolumn{6}{|l|}{ Histology } \\
\hline Ductal & 1.0 (reference) & 1.0 (reference) & 1.0 (reference) & 1.0 (reference) & 1.0 (reference) \\
\hline Lobular & $0.89[0.83,0.95]$ & $0.90[0.84,0.96]$ & $0.90[0.84,0.96]$ & $0.92[0.83,1.02]$ & $0.90[0.82,1.0]$ \\
\hline Other & $0.96[0.91,1.02]$ & $0.95[0.90,1.01]$ & $0.95[0.90,1.01]$ & $0.96[0.89,1.05]$ & $0.97[0.88,1.06]$ \\
\hline \multicolumn{6}{|l|}{ Extent of disease } \\
\hline Local & 1.0 (reference) & 1.0 (reference) & 1.0 (reference) & 1.0 (reference) & 1.0 (reference) \\
\hline Regional & $1.07[1.02,1.12]$ & $1.06[1.01,1.11]$ & $1.06[1.01,1.11]$ & $1.04[0.97,1.11]$ & $1.05[0.97,1.13]$ \\
\hline Distant & $1.41[1.29,1.53]$ & $1.34[1.22,1.47]$ & $1.33[1.21,1.47]$ & $1.34[1.12,1.60]$ & $1.25[1.07,1.47]$ \\
\hline Unknown & $1.02[0.92,1.12]$ & $0.96[0.86,1.06]$ & $0.96[0.86,1.06]$ & $0.97[0.81,1.17]$ & $1.03[0.84,1.27]$ \\
\hline \multicolumn{6}{|l|}{$\begin{array}{l}\text { Any treatment within } \\
12 \text { months }\end{array}$} \\
\hline No & 1.0 (reference) & & & & \\
\hline Yes & $1.07[0.91,1.26]$ & & & & \\
\hline \multicolumn{6}{|l|}{$\begin{array}{l}\text { Any surgery within } \\
12 \text { months }\end{array}$} \\
\hline No & & 1.0 (reference) & & & \\
\hline Yes & & $0.93[0.85,1.02]$ & & & \\
\hline \multicolumn{6}{|l|}{$\begin{array}{l}\text { Surgery within } \\
12 \text { months }\end{array}$} \\
\hline None & & & 1.0 (reference) & & \\
\hline Breast conserving & & & $0.92[0.84,1.01]$ & & \\
\hline Mastectomy & & & $0.94[0.86,1.03]$ & & \\
\hline \multicolumn{6}{|l|}{ Reconstruction } \\
\hline None & & & & & 1.0 (reference) \\
\hline Immediate & & & & & $0.49[0.41,0.58]$ \\
\hline 12 month & & & & & $0.42[0.34,0.51]$ \\
\hline
\end{tabular}


Table 3 (continued)

\begin{tabular}{|c|c|c|c|c|c|}
\hline \multirow[t]{2}{*}{ Independent variables } & \multicolumn{5}{|c|}{ Odds Ratio [95\% CI] Socioeconomic disadvantage } \\
\hline & $\begin{array}{l}\text { Model } 1 \text { (any treat- } \\
\text { ment) }\end{array}$ & Model 2 (any surgery) & $\begin{array}{l}\text { Model } 3 \text { (surgery } \\
\text { type) }\end{array}$ & Model 4 (BCS cases) & $\begin{array}{l}\text { Model } 5 \text { (Mastectomy } \\
\text { cases) }\end{array}$ \\
\hline 5 year & & & & & $0.61[0.51,0.75]$ \\
\hline \multicolumn{6}{|l|}{$\begin{array}{l}\text { Radiotherapy within } \\
12 \text { months }\end{array}$} \\
\hline No & & 1.0 (reference) & 1.0 (reference) & 1.0 (reference) & 1.0 (reference) \\
\hline Yes & & $0.95[0.91,0.99]$ & $0.95[0.91,1.00]$ & $0.90[0.83,0.97]$ & $1.01[0.93,1.09]$ \\
\hline \multicolumn{6}{|l|}{$\begin{array}{l}\text { Chemotherapy within } \\
12 \text { months }\end{array}$} \\
\hline No & & 1.0 (reference) & 1.0 (reference) & 1.0 (reference) & 1.0 (reference) \\
\hline Yes & & $1.04[0.99,1.09]$ & $1.04[0.98,1.09]$ & $1.09[1.01,1.16]$ & $0.94[0.87,1.02]$ \\
\hline \multicolumn{6}{|l|}{$\begin{array}{l}\text { Hormone therapy } \\
\text { within } 12 \text { months }\end{array}$} \\
\hline No & & 1.0 (reference) & 1.0 (reference) & 1.0 (reference) & 1.0 (reference) \\
\hline Yes & & $0.95[0.91,0.99]$ & $0.95[0.91,0.99]$ & $0.93[0.88,0.99]$ & $0.95[0.88,1.02]$ \\
\hline \multicolumn{6}{|l|}{$\begin{array}{l}\text { Immunotherapy within } \\
12 \text { months }\end{array}$} \\
\hline No & & 1.0 (reference) & 1.0 (reference) & 1.0 (reference) & 1.0 (reference) \\
\hline Yes & & $1.02[0.94,1.10]$ & $1.01[0.94,1.10]$ & $1.02[0.91,1.15]$ & $1.01[0.90,1.13]$ \\
\hline
\end{tabular}

NSW New South Wales, IRSD Index of Relative Socioeconomic Disadvantage, BCS breast conserving surgery, $C I$ confidence interval

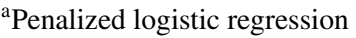

- Chemotherapy - higher odds for Model 4.

- Hormone therapy - lower odds for models 2-4.

\section{Residential remoteness}

From Table 4 it is evident that odds of residing in more remote areas were related to:

- Age at diagnosis - compared with $<40$ years, elevated odds for older ages in Models 1 and 4, and for 40-79 years in Models 2 and 3.

- Socioeconomic disadvantage - compared with most disadvantaged, progressively lower odds for lesser disadvantaged areas.

- Country of birth - compared with Australia, lower odds for mainly English-speaking countries and (more so) for mainly non-English countries in all Models.

- Stage (extent of disease) - compared with local, lower odds for regional and (more so) distant stage in Models $1-4$.

- Any treatment - lower odds in Model 1.

- Breast reconstruction (mastectomy cases) - compared with no reconstruction, lower odds for immediate reconstruction in Model 5.

- Radiotherapy - lower odds for all Models.

\section{Disease-specific survival}

\section{Unadjusted comparisons}

Differences in survival were very small by area of disadvantage albeit achieving statistical significance (Table 5). The 5 -year survival was $89 \%$ for the more disadvantaged areas compared with $90 \%$ for the less disadvantaged. Differences were smaller again and not statistically significant by residential remoteness, with a 5-year survival of $90 \%$ presenting (Table 5).

\section{Adjusted comparisons}

Multivariate competing risk regression showed an elevated risk of breast cancer death in women aged $70+$ years compared with $<40$ years, after adjusting for other characteristics in the model (Table 6). The more advanced the stage, the higher was the risk of death. Women born in mainly non-English speaking countries were less likely to have a recorded breast cancer death than the Australian born. There were also differences by histology type where, compared with ductal lesions, the risk of death was lower for women with lobular but higher for those with other histology types. A lower risk applied to 2008-2015 than in 2002-2007 diagnoses with sHRadjusted $=0.74$ [0.70, 0.79]. Differences in risk of breast cancer death were not 


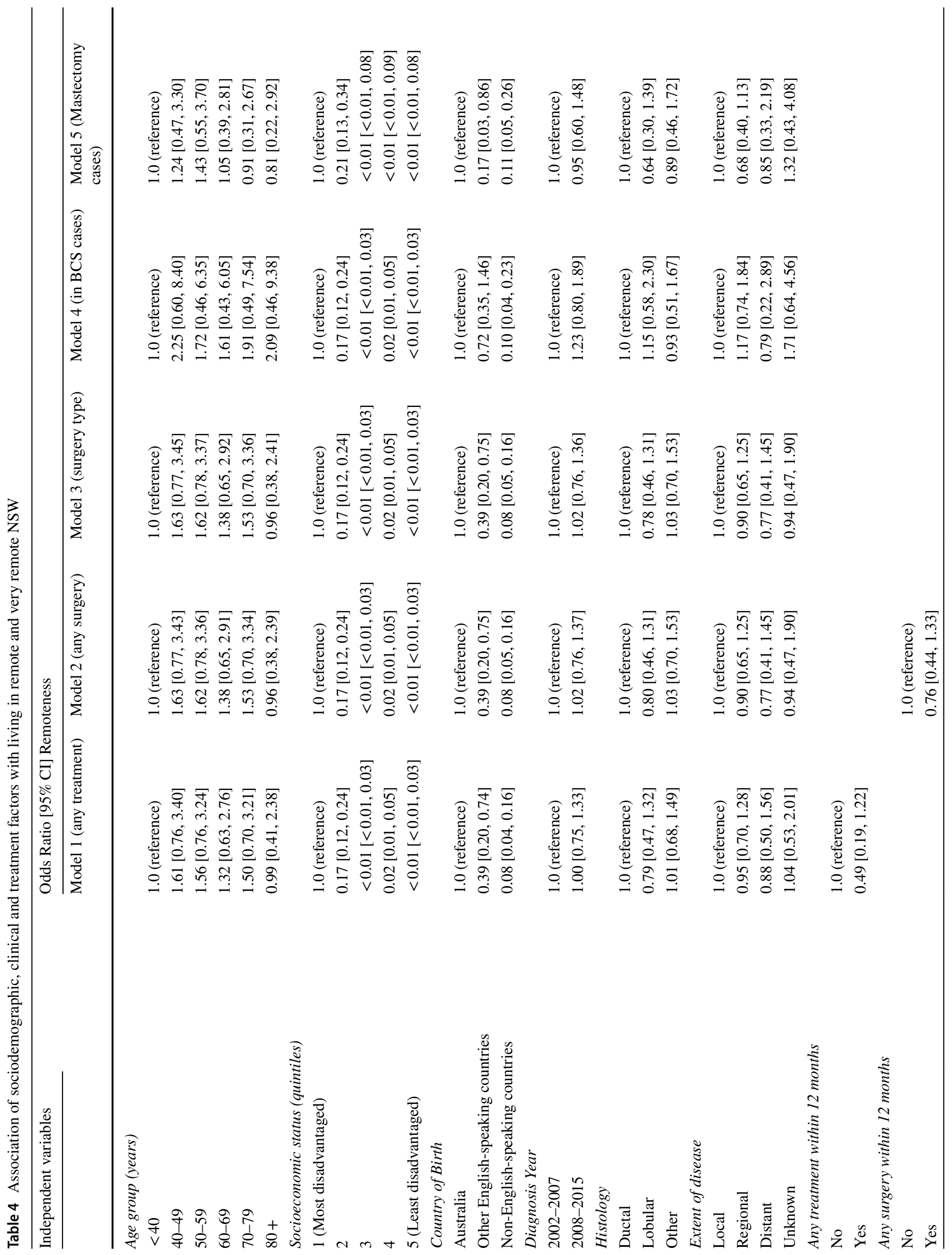




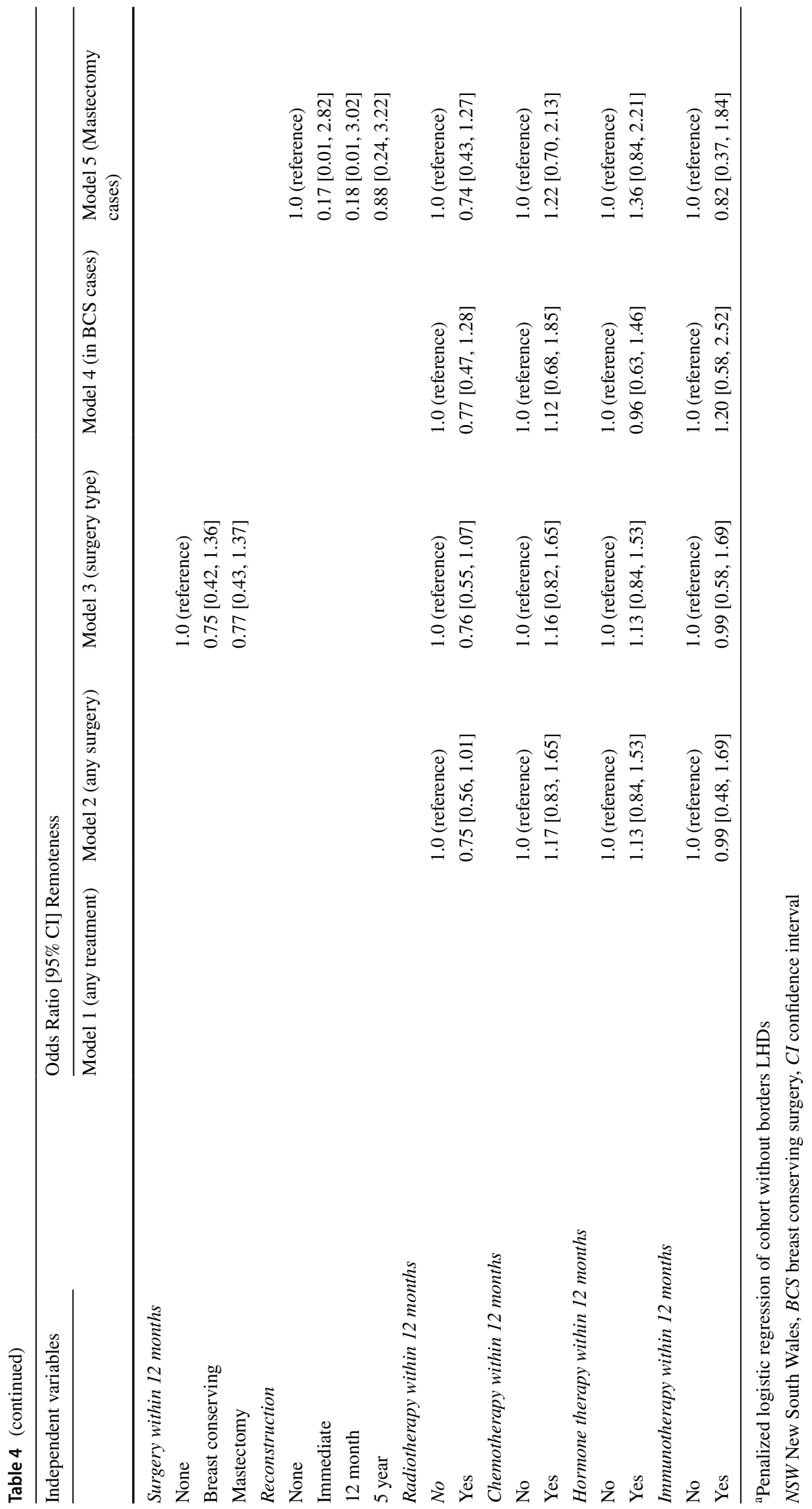


Table 5 Percentage KaplanMeier survival and 95\% confidence intervals

\begin{tabular}{lllll}
\hline $\begin{array}{l}\text { Years since } \\
\text { diagnosis }\end{array}$ & \multicolumn{2}{l}{ Socioeconomic status } & & \multicolumn{2}{l}{ Remoteness } \\
\cline { 2 - 3 } & Q1-Q2 & Q3-Q5 & M/IR/OR & R/VR \\
\hline 1 & $97.8[97.5,98.0]$ & $98.2[98.1,98.4]$ & $98.1[97.9,98.2]$ & $98.5[95.4,99.5]$ \\
2 & $95.5[95.1,95.8]$ & $96.1[95.9,96.3]$ & $95.9[95.7,96.1]$ & $97.4[93.9,98.9]$ \\
3 & $93.0[92.5,93.4]$ & $94.1[93.7,94.3]$ & $93.6[93.4,93.9]$ & $93.7[88.9,96.5]$ \\
5 & $88.6[88.1,89.2]$ & $90.4[90.0,90.8]$ & $89.8[89.5,90.1]$ & $90.3[84.5,94.0]$ \\
10 & $82.5[81.7,83.3]$ & $85.0[84.5,85.6]$ & $84.2[83.7,84.6]$ & $83.4[75.6,88.9]$ \\
\hline
\end{tabular}

$\mathrm{Q} 1-\mathrm{Q} 2=$ quintile 1-quintile 2; Q3-Q5 = quintile 3-quintile 5; M/IR/OR = metropolitan/inner regional/outer regional; $\mathrm{R} / \mathrm{VR}=$ remote/very remote evident by residential remoteness nor by socioeconomic disadvantage for four of the five quintiles. Compared with the most disadvantaged, however, the least disadvantaged had a lower risk of breast cancer death with sHRadjusted $=0.86$ [0.79, 0.94].

\section{Discussion}

The present results are reassuring in indicating a high fiveyear disease-specific survival from breast cancer at the high end of the international scale at $90 \%$ in New South Wales (NSW), and with evidence of an upward trend. These is little indication of differences in crude survival by residential remoteness despite large distances that many women have to travel for diagnosis and treatment and only a marginal difference by socioeconomic status. Equivalent adjusted survival applied in four of the five socioeconomic quintiles, but with the highest socioeconomic quintile showing higher survival.

Sub-hazard ratios indicated higher risks of death from breast cancer in older cases aged 70 years or more, and predictably, with more advanced stage (extent of disease), which is consistent with previous studies [2, 4]. While higher survival was found for women born in non-English speaking countries than for the Australian-born, confirmatory evidence is needed, given the potential for bias (e.g., from missed recording of deaths for women returning to their birth country with terminal disease) [10].

Adjusted analyses indicated more advanced stage in lower socioeconomic areas, with women from these areas presenting less evidence of breast reconstruction following mastectomy. Also, following breast conserving surgery, women from lower socioeconomic areas were less likely to have radiotherapy, and hormone therapy, but more likely to have chemotherapy.

Remoteness of residence was also associated in adjusted analyses with less radiotherapy and less use of immediate breast reconstruction. The lesser use of radiotherapy applied in all women, including those having breast conserving surgery. Contrary to earlier studies, however, remoteness of residence was not associated with lower survival or more advanced stage [2, 27].

Common to both the more socioeconomically disadvantaged and more remote areas was the lower exposure to radiotherapy and breast reconstruction. Adjuvant radiotherapy with breast conserving surgery is equivalent to mastectomy for overall, disease-free survival, and distant-metastasis free survival $[28,29]$. The lower likelihood of receiving adjuvant radiotherapy in breast conserving surgery cases with lower socioeconomic status may have contributed to the higher breast cancer survival seen in the least disadvantaged quintile.

Difficulties accessing radiotherapy services have been documented previously [30]. For example, some women from rural areas, particularly those of older age, may decline treatment recommendations due to the inconvenience of travelling, anticipated extended stays away from home, and limited social support available in their areas [31-33]. Greater use of hypofractionation protocols is occurring, however, which may lead to increased uptake of radiotherapy and concomitant reductions in mastectomies in clinical situations where otherwise breast conserving surgery/radiotherapy is an evidence-based option, including in women of lower socioeconomic status who may face more financial pressures through loss of work and family costs associated with attending radiotherapy [32,33].

Considerable effort and investment are being made to address access issues [34]. Cancer care centres in NSW are now readily accessible (within $100 \mathrm{~km}$ ) for $95 \%$ of the population [35]. The extent to which remaining access issues are deterring radiotherapy requires further research.

Reconstruction is preferred by many women to minimise impact on body image following breast cancer surgery [36, 37]. However, the proportion of women having this treatment following a mastectomy is low in NSW (and in Australia overall). Private health insurance and younger age are strong predictors of breast reconstruction [38]. This study found immediate reconstruction to be negatively associated with residential remoteness and strongly associated with higher socioeconomic status. 
Table 6 Sociodemographic and clinical predictors of breast cancer mortality in NSW breast cancer cases $(n=54,662), 2002-2015$

Subhazard ratios $[95 \% \mathrm{CI}]$

\begin{tabular}{|c|c|}
\hline \multicolumn{2}{|l|}{ Age group (years) } \\
\hline$<40$ & 1.0 \\
\hline $40-49$ & $0.80[0.66,0.99]$ \\
\hline $50-59$ & $1.03[0.85,1.24]$ \\
\hline $60-69$ & $1.18[0.97,1.42]$ \\
\hline $70-79$ & $2.16[1.79,2.60]$ \\
\hline $80+$ & $3.22[2.67,3.90]$ \\
\hline \multicolumn{2}{|l|}{ Remoteness } \\
\hline Metropolitan & 1.0 \\
\hline Inner regional & $0.98[0.92,1.05]$ \\
\hline Outer regional & $1.08[0.96,1.20]$ \\
\hline Remote \& very remote & $0.95[0.67,1.34]$ \\
\hline \multicolumn{2}{|l|}{ Socioeconomic status (quintiles) } \\
\hline 1 (Most disadvantaged) & 1.0 \\
\hline 2 & $1.04[0.96,1.13]$ \\
\hline 3 & $0.94[0.86,1.03]$ \\
\hline 4 & $0.95[0.87,1.04]$ \\
\hline 5 (Least disadvantaged) & $0.86[0.79,0.94]$ \\
\hline \multicolumn{2}{|l|}{ Country of Birth } \\
\hline Australia & 1.0 \\
\hline Other English-speaking countries & $0.97[0.86,1.08]$ \\
\hline Non-English-speaking countries & $0.80[0.73,0.87]$ \\
\hline \multicolumn{2}{|l|}{ Diagnosis year } \\
\hline $2002-2007$ & 1.0 \\
\hline $2008-2015$ & $0.74[0.70,0.79]$ \\
\hline \multicolumn{2}{|l|}{ Histology } \\
\hline Ductal & 1.0 \\
\hline Lobular & $0.83[0.73,0.94]$ \\
\hline Other & $1.14[1.05,1.24]$ \\
\hline \multicolumn{2}{|l|}{ Extent of disease } \\
\hline Local & 1.0 \\
\hline Regional & $5.19[4.54,5.94]$ \\
\hline Distant & $36.79[32.14,42.10]$ \\
\hline Unknown & $7.02[5.95,8.29]$ \\
\hline
\end{tabular}

Model included interaction terms with time for age group, histology, extent of disease and country of birth;

$N S W$ New South Wales, $C I$ confidence interval

1. Metadata Online Registry (2012) AIHW. https://meteor.aihw.gov. $\mathrm{au} /$ content/index.phtml/itemId/480010

Universal Health Insurance in Australia (Medicare) covers breast reconstruction costs in the private sector. Nonetheless, waiting times can be an issue in the public sector, such that public patients may be less likely than those in the private sector to obtain these services [39]. Women with comorbidity are less likely to have reconstruction which may contribute to the association of lower reconstruction rates with socioeconomic status, as seen in the present study [40, 41].
Further research is needed into cultural differences by socioeconomic status and remoteness that may also affect uptake of breast reconstruction following mastectomy. Meanwhile, promoting greater discussion between clinicians and patients regarding the option of breast reconstruction, and also of means of improving the acceptability of the procedure, may serve to minimise variation across sociodemographic groups $[42,43]$. As some women living remotely are more likely to have mastectomy due to barriers to receiving adjuvant therapy, it is important that opportunities for breast reconstruction are readily available to them.

Recent data show a lower cancer survival for people living in more socioeconomically disadvantaged areas [10]. We found breast cancer survival and associated factors largely align with other reports with lower breast cancer mortality occurring in those living in areas of least socioeconomic disadvantage at diagnosis $[44,45]$. The present data show little difference in survival by remoteness of residence, however, which is consistent with earlier research [46].

The strengths of this study are the-whole-of population approach to breast cancer analysis in NSW. Understanding real-world treatment patterns is important to interpreting the progressive increases in survival. Data linkage provides the opportunity to investigate treatment patterns at an all-of-health system level. Monitoring of differences in treatment, particularly for priority populations, are important to identify areas of unmet need for policy development.

Limitations of this study include the absence of biomarker data to enable assessment of the appropriate use of immunotherapies and hormone therapy. Other investigators are analysing Australian data on the patterns of use and outcomes for HER2 positive women, showing for example that women receiving trastuzumab are less likely to complete treatment if living in areas of socioeconomic disadvantage, or in remote areas [47]. The analysis of associations between treatment and remoteness were limited by the smaller population of women living in remote and very remote areas, which reduced the statistical power available to reveal differences.

\section{Conclusions}

Survival from breast cancer is high in NSW by world standards and increased further between 2002-2007 and 2008-2015. Little evidence of survival differences presents in adjusted analyses by remoteness but there is evidence that residents of areas in highest socioeconomic quintile have higher survival. Policy makers should continue their focus on initiatives to improve survival further by addressing barriers to cancer care, particularly as related to use of radiotherapy following breast conserving surgery and breast reconstruction following mastectomy, whether due to socioeconomic or geographic barriers. 


\section{Declarations}

Acknowledgements The authors are grateful to the NSW Ministry of Health for access to NSW data.

Author contributions Study concept: DR, DC. Study Design: DR, DC. Data acquisition: DR, DC. Interpretation of the data: DR, EE, MWS, SM, ZSK, SW, ML, DC. Manuscript writing: EB. Critical revisions: DR, EE, MWS, SM, ZSK, ML, DC. Data analysis: EB. All authors have read and approved the final version.

Funding Funding was from the Cancer Institute NSW with a contribution to the report writing also from the National Breast Cancer Foundation (CRP-17-001). Funding bodies had no role in the design, analysis, interpretation of data or in writing the manuscript.

Data availability The data that support the findings of this study are available from the Cancer Institute NSW and Australian Institute of Health and Welfare, but restrictions apply to the availability of these data, which were used under license for the current study, and so are not publicly available. However, data are available from the authors upon reasonable request and with permission of the NSW Cancer Institute and Australian Institute of Health and Welfare.

\section{Compliance with ethical standards}

Conflict of interest The authors declare that they have no competing interests.

Ethics approval and consent to participate The study was approved by the NSW Population and Health Services Research Ethics Committee (HREC/15/CIPHS/15). This followed a scientific review guided by the STROBE checklist for observational studies in epidemiology.

Open Access This article is licensed under a Creative Commons Attribution 4.0 International License, which permits use, sharing, adaptation, distribution and reproduction in any medium or format, as long as you give appropriate credit to the original author(s) and the source, provide a link to the Creative Commons licence, and indicate if changes were made. The images or other third party material in this article are included in the article's Creative Commons licence, unless indicated otherwise in a credit line to the material. If material is not included in the article's Creative Commons licence and your intended use is not permitted by statutory regulation or exceeds the permitted use, you will need to obtain permission directly from the copyright holder. To view a copy of this licence, visit http://creativecommons.org/licenses/by/4.0/.

\section{References}

1. Australian Institute of Health and Welfare (2020) Cancer data in Australia 2020. Australian Institute of Health and Welfare. https:// www.aihw.gov.au/reports/cancer/cancer-data-in-australia/conte nts/summary. Accessed 26 November 2020

2. AIHW (2019) Cancer in Australia 2019. vol Cat. no. CAN 123. AIHW, Canberra

3. AIHW (2016) Australia's Health 2016. AIHW, Canberra

4. Cancer Australia (2018) National Cancer Control Indicators: Distribution by stage. Cancer Australia. https://ncci.canceraustralia. gov.au/diagnosis/distribution-cancer-stage/distribution-cancerstage. Accessed 26 November 2020
5. Baade PD, Turrell G, Aitken JF (2011) Geographic remoteness, area-level socio-economic disadvantage and advanced breast cancer: a cross-sectional, multilevel study. J Epidemiol Comm Health 65(11):1037-1043. https://doi.org/10.1136/jech.2010.114777

6. Lyle G, Hendrie GA, Hendrie D (2017) Understanding the effects of socioeconomic status along the breast cancer continuum in Australian women: a systematic review of evidence. Int J Equity Health 16(1):182. https://doi.org/10.1186/s12939-017-0676-X

7. Mitchell KJ, Fritschi L, Reid A, McEvoy SP, Ingram DM, Jamrozik K, Clayforth C, Byrne MJ (2006) Rural-urban differences in the presentation, management and survival of breast cancer in Western Australia. Breast 15(6):769-776. https://doi.org/10. 1016/j.breast.2006.04.001

8. NSW Ministry of Health (2016) NSW Cancer Plan. Cancer Institute NSW, Sydney

9. Cancer Institute NSW (2002) Reporting for Better Cancer Outcomes Program. Cancer Institute NSW. https://www.cancer.nsw. gov.au/what-we-do/supporting-cancer-care/reporting-for-bettercancer-outcomes-program. Accessed 26 November 2020

10. Tervonen HE, Aranda S, Roder D, You H, Walton R, Morrell S, Baker D, Currow DC (2017) Cancer survival disparities worsening by socio-economic disadvantage over the last 3 decades in new South Wales, Australia. BMC Public Health 17(1):691. https://doi. org/10.1186/s12889-017-4692-y

11. Tervonen HE, Creighton N, Zhao GW, Ng M, Currow DC (2020) Capture of systemic anticancer therapy use by routinely collected health datasets. Public Health Res Pract. https://doi.org/10.17061/ phrp3012004

12. Public Health Act No 127 (2010) NSW, Australia

13. Cancer Institute NSW (2020) NSW Cancer Registry. Cancer Institute NSW. https://www.cancer.nsw.gov.au/research-and-data/cancer-data-and-statistics/request-unlinked-unit-record-data-for-resea rch/nsw-cancer-registry. Accessed 26 November 20

14. Metadata Online Registry (2012) AIHW. https://meteor.aihw.gov. $\mathrm{au} /$ content/index.phtml/itemId/480010

15. NSW Ministry of Health (2020) NSW Admitted Patient Data Collection. https://www.cherel.org.au/data-dictionaries\#section1. Accessed 28 November 2020

16. Australian Government DoH (2020) Medical Benefits Schedule. Commonwealth of Australia. https://www.aihw.gov.au/aboutour-data/our-data-collections/medicare-benefits-schedule-mbs. Accessed 28 September 2020

17. Australian Government DoH (2020) Pharmaceutical Benefits Scheme. https://www.aihw.gov.au/about-our-data/our-data-colle ctions/pharmaceutical-benefits-scheme. Accessed 28 September 2020

18. The Sax Institute (2020) Secure Unified Research Environment. https://www.saxinstitute.org.au/our-work/sure/. Accessed 28 September 2020

19. Australian Bureau of Statistics (2006) An introduction to SocioEconomic Indexes for Areas (SEIFA). Canberra

20. Australian Bureau of Statistics (2011) Australian Statistical Geography Standard (ASGS): Volume 5-remoteness structure. vol 5. Australian Bureau of Statistics, Canberra

21. Independent Hospital Pricing Authority (2020) ICD-10-AM/ ACHI/ACS, 8th edn. IHPA, Sydney

22. Devika S, Jeyaseelan L, Sebastian G (2016) Analysis of sparse data in logistic regression in medical research: a newer approach. J Postgrad Med 62(1):26-31. https://doi.org/10.4103/0022-3859. 173193

23. Heinze G, Schemper M (2002) A solution to the problem of separation in logistic regression. Stat Med 21(16):2409-2419. https:// doi.org/10.1002/sim.1047

24. StataCorp LLC (2019) Stata Version 16. Version 16.0 edn. StataCorp LLC, College Station, Texas, USA 
25. South Australian Cancer Registry (1999) Epidemiology of Cancer in South Australia. Incidence, mortality and survival 1977 to 1998. Government of South Australia, Adelaide

26. McGivern L, Shulman L, Carney JK, Shapiro S, Bundock E (2017) Death certification errors and the effect on mortality statistics. Public Health Rep 132(6):669-675. https://doi.org/10.1177/ 0033354917736514

27. Fine JP, Gray RJ (1999) A proportional hazards model for the subdistribution of a competing risk. J Am Stat Assoc 94(446):496509. https://doi.org/10.1080/01621459.1999.10474144

28. Australian Institute of Health and Welfare \& Cancer Australia (2012) Breast Cancer in Australia: an overview. vol CAN 67. Cancer Australia, Canberra

29. Fisher B, Anderson S, Redmond CK, Wolmark N, Wickerham DL, Cronin WM (1995) Reanalysis and results after 12 years of follow-up in a randomized clinical trial comparing total mastectomy with lumpectomy with or without irradiation in the treatment of breast cancer. N Engl J Med 333(22):1456-1461. https://doi. org/10.1056/NEJM199511303332203

30. van Maaren MC, de Munck L, de Bock GH, Jobsen JJ, van Dalen T, Linn SC, Poortmans P, Strobbe LJA, Siesling S (2016) 10 year survival after breast-conserving surgery plus radiotherapy compared with mastectomy in early breast cancer in the Netherlands: a population-based study. Lancet Oncol 17(8):1158-1170. https:// doi.org/10.1016/s1470-2045(16)30067-5

31. Hegney D, Pearce S, Rogers-Clark C, Martin-McDonald K, Buikstra E (2005) Close, but still too far. The experience of Australian people with cancer commuting from a regional to a capital city for radiotherapy treatment. Eur J Cancer Care 14(1):75-82. https:// doi.org/10.1111/j.1365-2354.2005.00525.x

32. Samarasinghe A, Chan A, Hastrich D, Martin R, Gan A, Abdulaziz F, Latham M, Zissiadis Y, Taylor M, Willsher P (2019) Compliance with multidisciplinary team meeting management recommendations. Asia Pac J Clin Oncol 15(6):337-342. https:// doi.org/10.1111/ajco.13240

33. Delaney GP, Gandhidasan S, Walton R, Terlich F, Baker D, Currow D (2016) The pattern of use of hypofractionated radiation therapy for early-stage breast cancer in New South Wales, Australia, 2008 to 2012. Int J Radiat Oncol Biol Phys 96(2):266-272. https://doi.org/10.1016/j.jirobp.2016.05.016

34. Kok DL, Chang JH, Erbas B, Fletcher A, Kavanagh AM, Henderson MA, Gertig DM (2006) Urban-rural differences in the management of screen-detected invasive breast cancer and ductal carcinoma in situ in Victoria. ANZ J Surg 76(11):996-1001. https:// doi.org/10.1111/j.1445-2197.2006.03917.x

35. Murphy C, Sabesan S, Steer C, Yates P, Booms A, Jones V, Simpson A, Clarke K, Eek R, Ashley D, Goldstein D, Packer C, Tuthill F, Boyce A, Underhill C (2015) Oncology service initiatives and research in regional Australia. Aust J Rural Health 23(1):40-48. https://doi.org/10.1111/ajr.12173

36. NSW Ministry of Health (2014) NSW Rural Health Plan: Towards 2021. NSW Ministry of Health, North Sydney

37. Chao LF, Patel KM, Chen SC, Lam HB, Lin CY, Liu HE, Cheng MH (2014) Monitoring patient-centered outcomes through the progression of breast reconstruction: a multicentered prospective longitudinal evaluation. Breast Cancer Res Treat 146(2):299-308. https://doi.org/10.1007/s10549-014-3022-7

38. Elder EE, Brandberg Y, Bjorklund T, Rylander R, Lagergren J, Jurell G, Wickman M, Sandelin K (2005) Quality of life and patient satisfaction in breast cancer patients after immediate breast reconstruction: a prospective study. Breast 14(3):201-208. https:// doi.org/10.1016/j.breast.2004.10.008

39. Bell RJ, Robinson PJ, Fradkin P, Schwarz M, Davis SR (2012) Breast reconstruction following mastectomy for invasive breast cancer is strongly influenced by demographic factors in women in Victoria, Australia. Breast 21(3):394-400. https://doi.org/10. 1016/j.breast.2012.04.006

40. Cancer Australia (2020) Comparison of public versus private breast reconstruction surgery. Cancer Australia. https://www. canceraustralia.gov.au/affected-cancer/cancer-types/breast-cancer/ treatment/surgery/breast-reconstruction/deciding-about-breastreconstruction/factors-affecting-0. Accessed 23 September 2020

41. Hall SE, Holman CD (2003) Inequalities in breast cancer reconstructive surgery according to social and locational status in Western Australia. Eur J Surg Oncol 29(6):519-525. https://doi.org/10. 1016/s0748-7983(03)00079-9

42. Reuben BC, Manwaring J, Neumayer LA (2009) Recent trends and predictors in immediate breast reconstruction after mastectomy in the United States. Am J Surg 198(2):237-243. https://doi. org/10.1016/j.amjsurg.2008.11.034

43. Chen JY, Malin J, Ganz PA, Ko C, Tisnado D, Tao ML, Timmer M, Adams JL, Kahn KL (2009) Variation in physician-patient discussion of breast reconstruction. J Gen Intern Med 24(1):99-104. https://doi.org/10.1007/s11606-008-0855-y

44. Retrouvey H, Zhong T, Gagliardi AR, Baxter NNMDP, Webster F (2019) How patient acceptability affects access to breast reconstruction: a qualitative study. BMJ Open 9(9):e029048. https:// doi.org/10.1136/bmjopen-2019-029048

45. Cramb SM, Mengersen KL, Turrell G, Baade PD (2012) Spatial inequalities in colorectal and breast cancer survival: premature deaths and associated factors. Health Place 18(6):1412-1421. https://doi.org/10.1016/j.healthplace.2012.07.006

46. Lao C, Lawrenson R, Edwards M, Campbell I (2019) Treatment and survival of Asian women diagnosed with breast cancer in New Zealand. Breast Cancer Res Treat 177(2):497-505. https://doi.org/ 10.1007/s10549-019-05310-z

47. Taylor R (1997) Breast cancer five-year survival, by New South Wales regions, 1980 to 1991. Aust N Z J Public Health 21(2):206210. https://doi.org/10.1111/j.1467-842x.1997.tb01684.x

48. Tang M, Schaffer A, Kiely BE, Daniels B, Simes RJ, Lee CK, Pearson SA (2020) Correction: Treatment patterns and survival in HER2-positive early breast cancer: a whole-of-population Australian cohort study (2007-2016). Br J Cancer. https://doi.org/10. 1038/s41416-020-0908-5

Publisher's Note Springer Nature remains neutral with regard to jurisdictional claims in published maps and institutional affiliations. 